\title{
Oaklisp: an Object-Oriented Scheme with First Class Types
}

\author{
Kevin J. I ang and Barak A. Pearlmutter \\ Department of Computer Science \\ Carnegie-Mellon University \\ Pittsburgh, PA 15213
}

\begin{abstract}
The Scheme papers demonstrated that lisp could be made simpler and more expressive by clevating functions to the level of first class objects. Oaklisp shows that a message based language can derive similar benefits from having first class types.
\end{abstract}

\section{Introduction}

Oaklisp is a message bascd, multiple inheritence dialect of lisp. Programs are written using lisp syntax. and traditional lisp data types coexist with a Smalltalk style class hicrarchy. This paper assumes that the reader is familiar with one of the many object-oriented lisp dialects of this sor, and will therefore concentrate on the unique aspects of Oaklisp which are mostly due to the influcnce of Scheme.

Oaklisp is based on Scheme in two ways. Scheme was used as the model for syntactic details whenever possible in order to minimize our contribution to the continual proliferation of incompatible varieties of lisp. More significantly, Oaklisp is based on the Scheme philosophy. which states that the primitive forms of a language should be simple. powcrful, and meaningful from several points of view. The careful design of Oaklisp permits its object-oriented and procedural sides to be more closcly integrated than in a language which just hangs a separate message facility on the side of an existing lisp. Nlthough Oaklisp is object-oriented from the core, all of its features bchave in such a way that pure Scheme emerges as an alternate programming style.

Because Oaklisp is so closely related to Scheme, it is worth taking a look at the main ideas of Scheme before procceding. The conceptual foundation of the language is that functions are objects just like everything else, which means they can be returned from calls, passed around, stored in data structures, and so forth. This principle has

\footnotetext{
'This work was supported by grants fiom DARPA and the System Development J.oundation Barak Pcarlmutter is a llertz Fellow.
}

Permission to copy without fee all or part of this material is granted provided that the copies are not made or distributed for direct commercial advantage, the ACM copyright notice and the litle of the publication and its date appear, and notice is given that copying is by permission of the Association for Computing Machinery. To copy otherwise, or to republish, requires a fee and/ or specific permission.

(c) 1986 ACM 0-89791-204-7/86/0900-0030 75t several implications that are not immediately obvious. Because a function can be applied at a point distant in time and space from its point of origin, it must be able to remember the bindings of any variables that were visible when it was made. This additional complexity is offset by the ability to write many previously primitive control structures at the user level, and by the fact that the special mechanisms that lisp ordinarily uses for defining and applying functions can be dispensed with.

In lisp, the car position of a function call is treated as the name of a function which is looked up in a special table and then applied to the values obtained by evaluating the arguments of the call. In Scheme, the car of a call is an evaluated position. Although any expression can occur in the car, it is common for the expression to be a variable, in which case a call looks exactly like it would in lisp even though something completcly different is going on. For example, the Scheme form (PLUS 12 ) is cvaluated by looking up the binding of the variable PLUS and applying the resulting function to the values 1 and 2. Because functions are manipulated using the same mechanisms as other forms of data, they are first class. Because functions are never found by looking up their name, they are anonymous. It is worth pointing out that when a function is cummonly bound to a particular variable (such as pLUS), it is convenient to speak as if the variable's name were the function's name. This practice should not be allowed to obscure the fact that the function is really an anonymous object which happens to be accessible through a standard variable binding.

The Oaklisp version of a function call is an amplified version of what we have just seen in Scheme, with identical syntax and closely related semantics. The first step in the evaluation of a call is the same. namely the recursive evaluation of the subelements of the form. The message based semantics of Oaklisp only becomes manifest in the application step of evaluation, where the car value is taken to be an operation and the second value is taken to be an object whose type determines the method which is invoked to perform the operation. The remaining arguments are passed along to the method, but play no role in its selection. It should be clear that this message passing paradigm is basically the same as in Smalltalk. The inheritance and shadowing of methods occurs in the usual way. The only major difference between Oaklisp and Smalltalk messages is that Oaklisp operations are not 
symbols; they are anonymous objects that may be passed around and compared. The anonymity of operations is necessary so that Oaklisp will have the correct Scheme semantics when a functional programming style is adopted. However. an operation is not a function by itself, since it is not directly associated with any code. $\Lambda \mathrm{n}$ operation is just a thing with a unique identity that in conjunction with a type specifies a method which can actually be executed.

To make all of this a bit more concretc, consider the cvaluation of the call (PLUS 23 ). The first subform is a variable which is dereferenced, yiclding an operation. The other two subforms are constants, so) they evaluate to themselves. The type of the distinguished first argument is retrieved, and then the method tables of the integer type and its supertypes are scarched using the anonymous operation as a kcy. If the tables have been set up correctly, the scarch leads to the selection of a method that knows how to add things to an integer. Finally, the method is invoked with the arguments 2 and 3.

We have scen how the cvaluation of calls is moderated by the type system, and in particular by the method tables of types. The ability to configure thesc tables is provided by the ADO-ME IHOO special form. For example, by cvaluating the following expression we can define a PLUS method for cons cells such that (PLus (CONS 23 )) $\rightarrow 6$.

(ADD-ME THOD (PLUS (PAIR) SELF)

(TIMES (CAR SELF) (CDR SELF))

This form tells the system to assixiate the method specified by the body with the operation plus in the method table of the type paIr. It is a special form rather than a call because of the keyword ADO-ME THOD and because the body and argument list (SELF) are not evaluated. However, the operation and type positions are evaluated, which means that plus is just a variable which is bound to the same opcration which will later be used as a method selector during a call.

We have repeatedly said that operations are things that can be passed around and stored in variables. The reader may wonder where operations come from in the first place. Since they are objects just like everything else, they are obtained the same way as any other object, namely by instantiating a type. An instance of a type is generated by sending the type a make message. For example, the system plus operation is defined by evaluating

(SETI PLUS (MAKE OPERation)).

Now according to the Oaklisp evaluation rules for calls, Operariom is not the name of a type but is a variable bound to a type object. Since types are anonymous things just like operations, they are obtained in an analogous manner, by instantiating the type trpe. For example, we could create a frog type with the following expression.

(SET! FROG (MAKE TYPE (LIST - AGE 'COLOR) (LIST OBJECT)))

In this case the maxe message takes extril arguments which specify the new type's instance variables and supertypes. Because the make expression is a call and not a special form, all of its subexpressions are evaluated. Observe that the first argument expression has been written in a manner that evaluates to a list of symbols. and that the second yiclds a list of type objects.

The type specificd as the supertype of I ROG is OBJeCr, which is the distinguished type that lives at the tup of the inheritance graph. Becausc every type is a subtype of o8jecr, a methend which has been installed in OBJt $\mathrm{Cl}$ will work on any ubject whatsocver and can be used as the default method for an operation. This ability to define default methods suggests the following strittegy for setting up a type predicate.

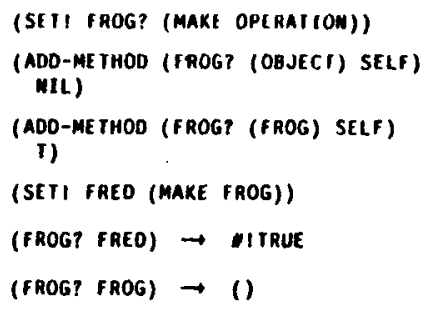

The last two expressions illustrate the fact that while FRED is a frog, IROG is a type. FRED and iROG are also objects, because FROG and TYPE are subtypes of the type 08Ject. These and other amaring facts can be seen in figure 1, which shows the primordial type hicrarchy together with the frog example. It is important to understand the difference between the is-a and subtjpe of relations. Whereas every object is the bottom of an is-a link, types are the only participants in subtype of links.

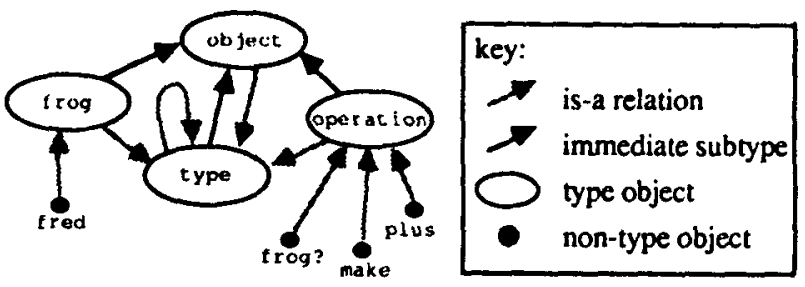

Figure I: The Primordial Types

Although the primordial types obJeCI, TYPE, and Operation may secm to be magic. they are just like any other types and could be defined at the user level. ${ }^{2}$ The general lick of magic in the type system permits a degree of openness and extensibility beyond that of Smalltalk and /ecial isp. both of which have lots of special machinery laying around. The later sections of this paper present several nifty features that can be defined in Oaklisp at the user level.

But first, there are a couple of details that need to be addressed in the frog example. The rrog type possesses instance variables which should be initialized when a frog is made. Since this is a common requirement. the make method for types sends new instances an

\footnotetext{
${ }^{2}$ Actually. some pointer tweaking is needed because of the circularities in their definitions:
} 
InIJialize message before returning them. The default ImItiatize method is a no-op. By specifying an initialize method for frogs, we can shadow out this default method and cause something uscful to happen.

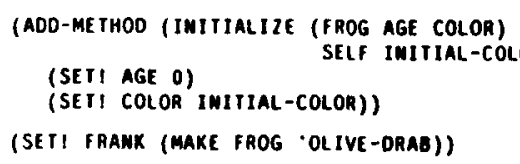

Notice that when an instance variable is used in the body of a method, it must be declared at the top of the method. This helps to disambiguate variable references for both the compiler and the programmer.

A particularly important aspect of the ADD-ME rHOO form is that the method is closed in its lexical environment when the form is evaluated. Together with the fact that AOD-ME THOO returns its operation argument, this rule allows the LAMBDA special form of Scheme to be defined with the following macro.

(LAMBDA arg-list. body) $\equiv$ (AOD-METHOD ((MAKE OPERAIION) (OBJECT). or -list). body)

When a LAmbon form is evaluated, it generates a new anonymous operation and supplies a default method for the opcration. Since the same piece of code is invoked every time the operation is sent in a message, the opcration behaves cxactly like a function. Using the LAMBOA form, Oaklisp programs can be written in a functional style that is indistinguishable from Scheme. In practice, programs tend to be written in a mixture of the functional and object-oriented styles. It is casy to combine the (wo) styles in a harmonious manner because the Scheme component of the language is just the natural result of having anonymous operations and a lexically scoped AOD-ME THOD form.

\section{The Oaklisp Cons Hlierarchy}

In the Oaklisp kernel, conses are defined in Oaklisp itself in a way open to extension by ordinary users. The cons hicrarchy (see figure 2) is rather detailed. allowing each method and subtype to be defined at the right level of abstraction.

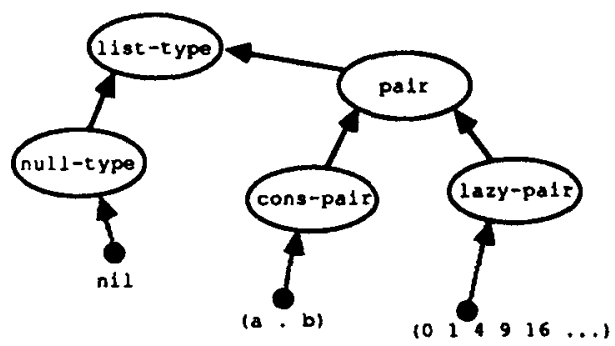

Figure 2: The Cons Hierarchy
(SFII LIST-TYPE (MAKE TYPE $\cdot()$ '(l))

(SETI MULL-TYPE (MAKE TYPE ' () (LIST LIST-TYPE OEJECT)))

(SETI PAIR (MAKE TYPE ') (L) (LST LIST-TYPE)))

(SETI COMS-PAIR (MAKE tYPE '(THE-CAR THE-CDA) (LIST PATR ODJECT)))

(ADO-NETHOO (CAR (CONS-PAIR the-CAR) SELF)

THE-CAR)

The paIr type is never instantiated: it is an abstract type for "things that behave like lisp conses." Methods for printing and mapping are defined at the palr level and are shared by all of paig's subtypes, while the subtypes themselves are responsible for handling CAn and con messages. Ordinary cons cells are instances of the type cons-paln, but other uscful subtypes of pair can be defined as well. For example, the following program fragment sets up a type of lazy pair that only computes its car and cdr when they are actually needed.

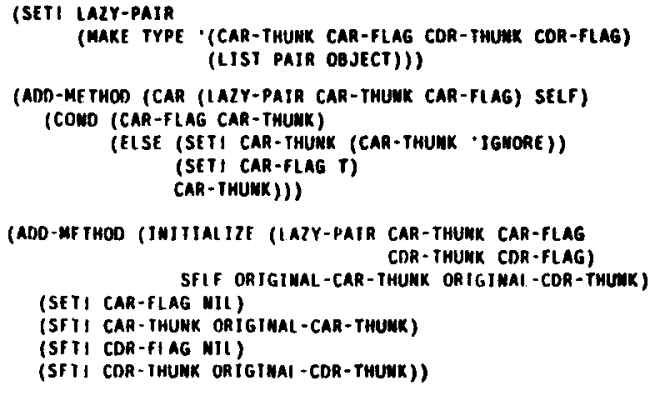

When we make a lazy-pair, we give it "thunks" for the car and cdr values. 'The pair then uses a call by need strategy, in which a thunk is used $w$ compute the car or cdr on first request, and the computed value is stored and returned immediatcly on future requests. The case with which we can create thunks is a consequence of the Scheme scoping rules, which allow us to close a function in the environment of its creation and to use the function even after the environment in which it was created has bcen exited. When we want to make a thunk for a computation, we just close a function to compute the needed value in the appropriate environment. For instance, to create an infinite list of squares we can write

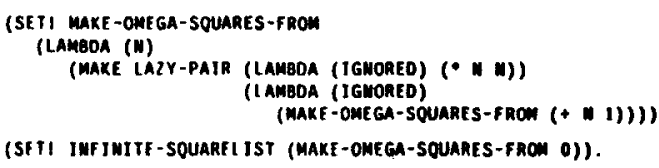

The syntax here is somewhat awkward ${ }^{3}$ but syntax is not the point of the example. We have created an infinite list which is computed on demand. Since we've built on the abstract pajk type, the list can be printed and manipulated like any other list; our lazy pairs deal with can and cDr a little ideosyncratically, but that's invisible from outside the type. For instance, if we were to now print imf imste-squaret ist, "( 10 1 14 - 18283648 ...)" would appear on our screen. It is interesting to note

\footnotetext{
${ }^{3}$ Expert lisp programmers will recogize the opportunity to definc a iazy-cous macro.
} 
that the printer normally abreviates long lists. printing "..." after a certain number of elements have been printed out. This feature is inherited by all subtypes of pair, so we don't have to do anything special to make lazy pairs print reasonably. This illustrates the usefulness of abstract types and the importance of separating them from particular implementations. It also shows the usefulness of defining very general methods at high levels of abstraction, as such a policy leads to greater code sharing.

It is equally easy to make a list that is overlayed onto a fractal set of points on the screen or a string that is the mapped image of a file. These examples illustrate the synergy between the dual linguistic paradigms embodicd in Oaklisp. The object orientation of Oaklisp allows us to write modular definitions that can be hooked right into the type hicrarchy, providing the user with a paletue of types having unifonn behavior but varying implementations. The Scheme semantics of Oaklisp allows these implementations to play games with higher order functions, using deviant Conniver-style control structures of the son that provided the original motivation for developing Scheme and Prolog.

\section{Coercable Types}

In this section we define a new metatype, coercable-trpe, whose instances are types that possess coercion operations. In order to coerce an object to one of these types, we send a message $w$ the type asting it to return its coercion operation, and then we apply the resulting operation to the object. For example, we might want to define a new kind of table that associates keys and values like a hash table but is implemented using a self-adjusting binary trec. By evaluating the expression

(SET) SLEATOR-TABLE (MAKE COERCABLE-TYPE '(ROOT SIZE) (LIST TABLE OBJECT)))

we can create the type and have a coercion operation generated automatically. To define methods for this operation, we can get our hands on it by sending the sleator-table type a coercer message. Then, if $r 00$ is bound to a table of some other sort, we can coerce it to a sleator-table by evaluating

((COERCER SLEATOR-TABLE) FOO).

Now that we have seen how coercable types should behave, we can actually define them. Observe how the anonymity of operations and types permits us to use type objects to govern access to coercion operations. First we create the new metatype with

(SEI) COERCABLE-IYPE (maKe TYPE '(COERCSON-OP) (LIST TYPE)))

This new metatype is just like the original metatype trpe except for a new instance variable coercion-op. Next we define the interface to Cocrcable types: the correER operation.

(SETI COERCER (MAXE OPERATIOH)
(ADD-MT THOO) (COFRCTA (COFRCAAIT - IYPF COEAGION-OP) SELF) Colncion-op)

It remains to define an initial in method for cocrcable types so that when one is created it will make itsclf a cocicing operation and stash it in its cofacroos-or instance variable.

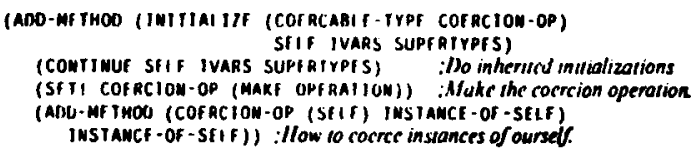

The expression (SET! COERCiOn-OP (MAKE OPEaATion)) is straightforward, but the other two forms in the initialization code requirc some explanation. The first form, (cowrinue ...), runs handlers for the inI Ijal itf operation that lie above the current point in the type hicrarchy. The code following the (comrrwuf ...) is execuled after these handiers, much like a Zccalisp : afyfa method. The cxpression (AOD-af thoo (COERcen (SfLf) ...) ...) adds a handler to the type that is being created. The handler is for the type's coercion operation, so this form tclls instances of the typc how to be cocrced to that type. Since they are already of the right type, all they need do is return themselves.

To give a concretc example of how this facility is used, imagine that we want to add complex numbers to our language, and that we want our complex numbers to have two different representations, cartesian and polar. We'd also like to be able to switch between representations conveniently. Our implementation will use an abstract type compcex and two concrete subtypes.

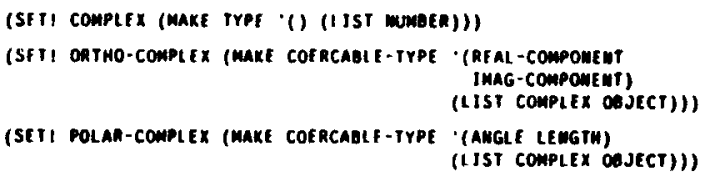

Now that we have some new types of numbers, we have to make them do all the things numbers are supposed to: addition, exponentiation, printing themselves, etc. The details are tedious; for expository purposes, a few examples suffice.

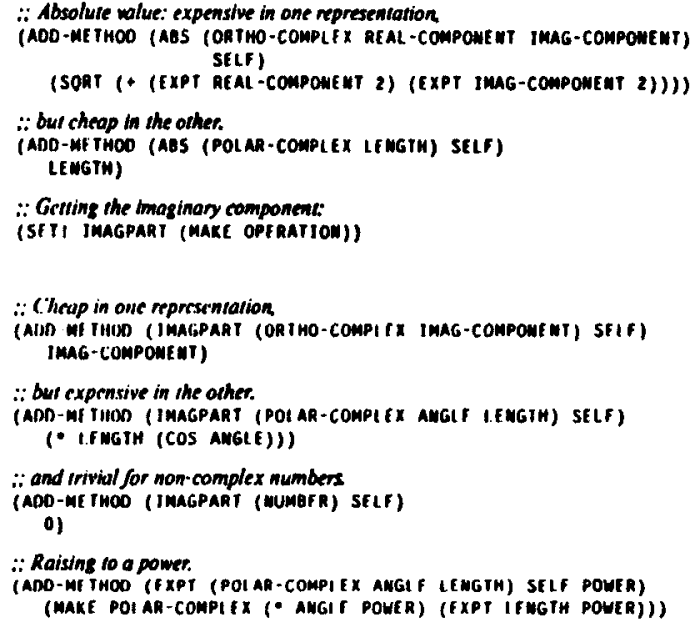




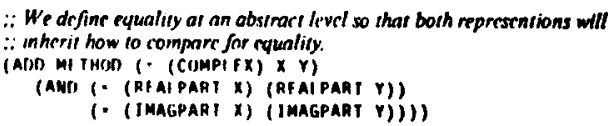

Now we get to usc our powerful cuercion operations. liach kind of complex number automatically knows huw to coerce to itsclf: we just have to tell each kind how to be cocreed to the other.

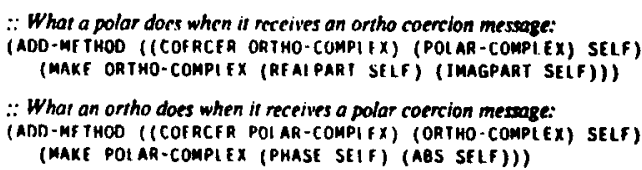

With everything defined, we're ready to demonstrate. Lets create a complex number represented in cartesian terms and coerce it into one represented in polar terms.

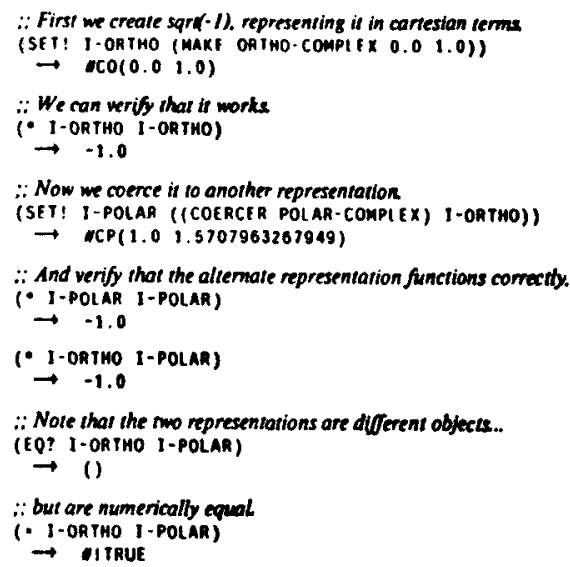

An interesting consequence of this coercable type mechanism is that we can try to coerce one thing to the type of something cise withoit knowing what that sccond type is. For example, suppose roo is a hash table and Bar is some kind of table that scems very fast and cficicient and we'd like to cocree foo into a table of the same sort. We get enR's type. ask it for its cocrcion uperation. and apply that operation to roo, as follows:

((COFACEA (GET-TYPE BAR)) FOO).

The contrast between our coercable type construction and Smaltalk class variabies is also interesting. In Smalltalk, there are spocial variables which are global to an entire type. The cofrctow-op instance variable of each coercable type is morally equivalent to a class variable, since any instance of a coercable type could get to the coercable opcration by running up its is-a link. By defining an interface at the type level allowing the variable to be accessed and sume macros to sugar the syntax, we could use this sort of definition to make things that look almost exactly like class variables. $\Lambda$ II of this activity can take place at user level-no modification or knowledge of system internals is necessary.

\section{Mixin Managers}

Frequently, type hierarchies become so rich that they threaten to overwhelm users with a plethora of possible combinations of mixins. The combinatorial explosion of the number of possible concocted types seems intrinsic to the style of programming involving multiple functionally orthogonal mixins. Above a certain level of complexity, finding a type with certain known characteristics can become difficult. Programmers are left wondering "Has a type based on foo with bar, baz and zonk mixcd in been creatcd, if so what's its name, and if not what should I name it and where should I define it?"

In Oaklisp, it is easy to define mixin managers that take care of this problem. When programmers need "the type based on foo with bar. baz and zonk mixcd in," they ask a mixin manager for it. If such a type has already been created. it is returned; if not, the mixin manager creates an approprinte new type, caches it, and returns it. This relieves programmers of the burden of remembering which types have been concocted and what they are named.

It is enlightening to examine the :mIxrupr defllavor option added to the flavors system in Symbolics 7ctal isp Relcase S. Although : HIxtune provides functionality vagucly similar to that of an Oaklisp mixin manager, the inplementation of :mI xiurf required major additions to the decply internal definitions of DFFFiavor and other portions of the flavor system, and was far from being a user level extension. The reason : mixturt was so difficult to define is that Zetalisp flavors are not first class. In Oaklisp. on the other hand, mixin managers are defined at user level.

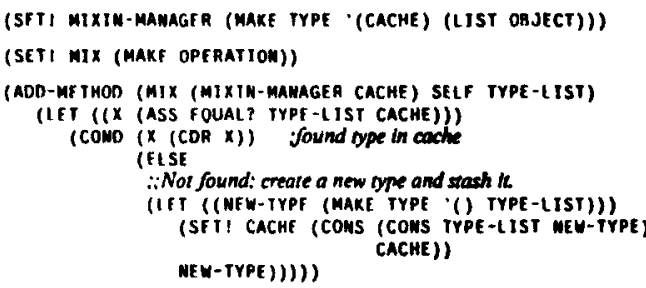

When an instance of the wIx In-nanaGer type receives a wIx message it gets one argument: a list of types to be mixed tugether. The mixin manager checks its cache, creating and caching the requested type if necessary.

To demonstrate a mixin manager in action, consider the Oaklisp operation hierarchy, which is quite elaborate. Some of the types and mixins involved are operation, OPEN-CODABLE-OPERATION, settagle-operation, locatagle-operatiom, tagtrapasle-mixin, and COMSTANT-Folongl.E-MIXIw. Their precise functionality isn't relevent; what is of note is that when we make a new opcration which should be a combination of a number of these types, we can use a mixin manager to

\footnotetext{
The Scheme features or Oaklisp provide ancther way to allow a number of methods to share a variable of their own. even if the methods are for different types, but this worts only if the method definitions share a single lexical scope.
} 
hclp us. We proceed by first making a mixin manager and then using it to get a complex combination of types.

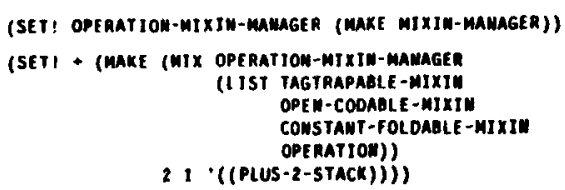

This definition of + is actually drawn from the Oaklisp kerncl, and is just like the definition of a regular operation. except for some extra mixins and initialization arguments. The mixins tell the compiler and runtime system special things about the operation, such as how to open code it in compiled code and what to do if the corresponding primitive instruction encounters a tag trap. The lucalization of all information about various aspects of addition in the addition operation itself is a great boon to modularity. Contrast this with the approach that is necessary in most systems, where information about addition is distributed between the operator itself, the compiler internals, and the runtime system. The clean interface to the runtime system and compiler in Oaklisp is made possible by the anonymity of the objects and the gencral type hierarchy.

\section{Semantic Foundations}

Types represent sets of objects. This relationship may be specified by a mapping $m$ which sends a type to the set of objects that it represents. In Oaklisp, types are themselves objects, so $m$ is actually a partial mapping from objects to sets of objects. Figure 3 is a Venn diagram that depicts the mapping $m$ and its interaction with the subtype predicate defined by the type system and the actual subset relation among sets of objects. The right hand side of the figure illustrates multiple inheritance, and the COERCABte-Trpe stuff shows how new metatypes fit into the system.
The mapping in may defined more formally as follows. I.et $O$ be the set of all objects. and $T$ the set of all type objects. Let $m: r \subseteq O \rightarrow 2^{o}$ be defined by $x \in m(a)$ if and only if (15-a? $x a$ ). Interestingly, $T=m$ (trpr) and hence irpr $\in m$ (rrpe) since (IS-A) irpe irpe). Such near circularitics raise a concern: when dealing with a system some of whose elements are members of scts represented by other elements, Russcll's paradox may result.

In order to allay concerns of this sort, it is necessary to prove that the type heirarchy in Oaklisp cannot give rise to contradictions. Such a proof requires an axiomatic formalization, so) that tools of logical analysis may be bruught to bear. A beneficial side effect of such a formalization is that it serves as a succinct and precise way to express the semantics of the type heirarchy. $A$ bit of the formalization is therefore presented here. We assume that the user will not redefine the primitives used. We let $a$ and $b$ range over types while $x$ ranges over all objects. The notation $a \leq b$ is used for (subrypt? $a b$ ).

$x \in m($ osject $)$.

The relation $\leq$ is a partial ordering of $T$.

If $a \leq b$ then $m(a) \leq m(b)$.

(GET-TYPF $x) \in T$.

If $x \in m(a)$ then (GET-rrpe $x$ ) $\leq a$.

$(\max a \quad \ldots) \in a$.

$a=($ GET-TYPE (MAKE $a$... $))$.

If $a$ is an clement of the list $l$ then (make trpe $l \ldots, \leq a$.

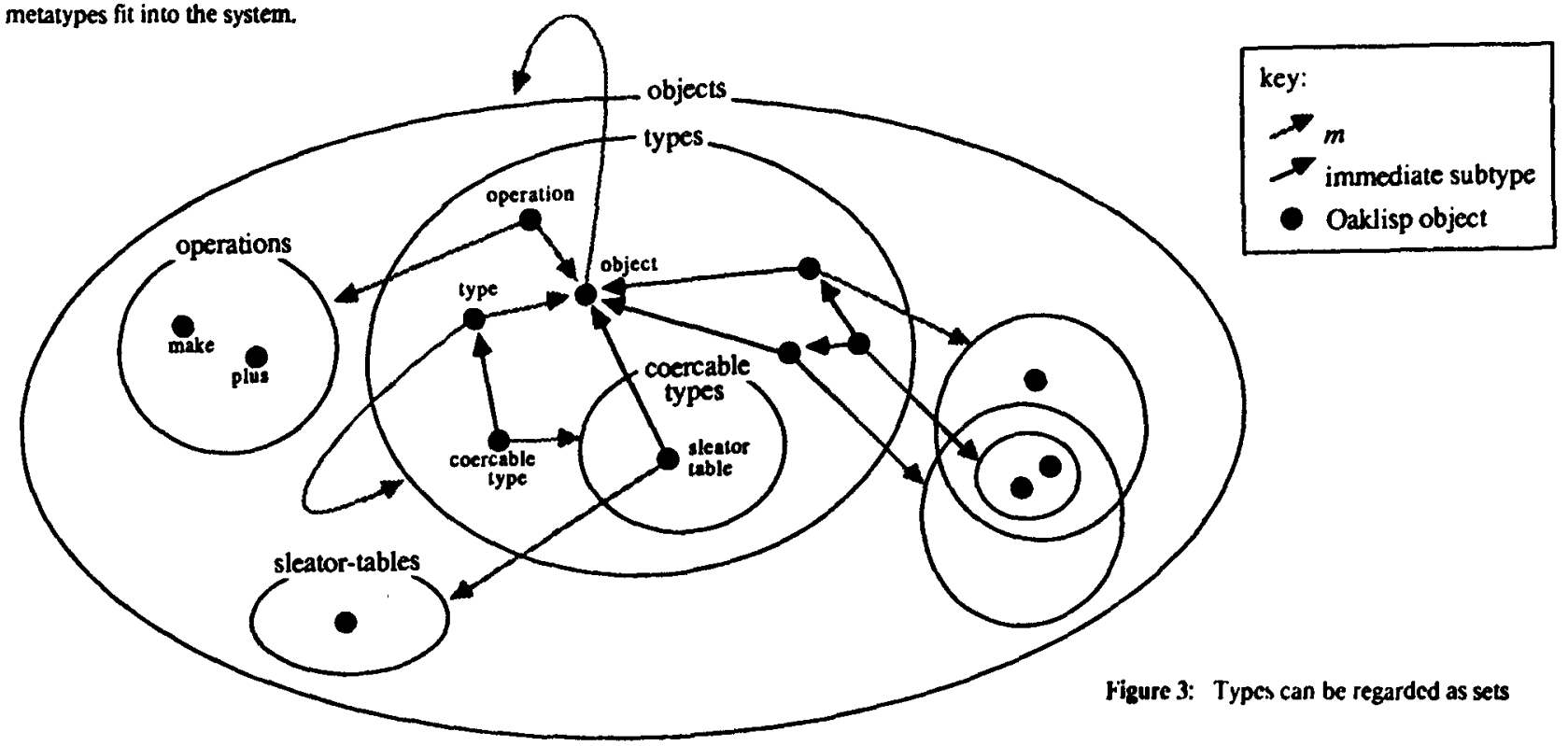


It is noteworthy that the GET-Trpe function can be formalized in this way. The implementation of GeT-trpe returns the contents of the type field of an object. Formally. Gr r-Trpe returns the smallest type containing the object it is applied to. The guarantee that such a smallest nontrivial type exists for every object in the system is one way in which the theory of the Oaklisp type heirarchy differs from usual set theory, and was necessary for our proof of consistency.

\section{Comparison to Other Work}

Oaklisp derives its message sending syntax from $T$, a highly developed dialect of Scheme. However, $T$ is not object-oriented in the usual sense since types are not visible to the user, there is no inheritance hicrarchy, and it is impussible to add new methods to existing objects.

Object I.isp, Common Losps, and New Flavors also use the T syntax for messages. Oaklisp differs from these languages in that its type hicrarchy lies at the heart of the language, climinating the distinction between "regular lisp stuff" and "object-oriented extension stuff." More importantly. Oaklisp's types are first class and can be meaningfully manipulatcd by user code.

\section{Conclusion}

We stated at the beginning of the paper that the unique features of Oaklisp are mostly due to the influence of the Scheme philosophy. For example, the tight coupling between the object-oriented and functional sides of Oaklisp is motivated by the principle of not creating two primitive mochanisms when one will suffice. Scheme also supplied the idea of first class functions, whose power can be exploited even when the message based aspects of the language are being to used to maintain a conservative module discipline. The usefulness of first class functions inspired the first class types of Oaklisp, which tum out to have similar benefits, making it easy to define meta tools which deal with the semantic substrate of the language itself. In some ways, Oaklisp's ability to manipulate its own type structure is analogous to the ability of 3-lisp to reflect upon its own control structure.

\section{Appendix: Current Implementation}

The implementation of Oaklisp is heavily influenced by $T$, which was designed as a systems programming language. In particular, the Oaklisp compiler differs from those written for instructional dialects of Scheme by emphasizing run-time efficiency rather than hooks for the debugger. For instance, appropriate castts forms are compiled as tight loops that do not generatc lambdas. This orientation also led us to provide for low-level access to native machine resources. Finally, $T$ provided the inspiration for Oaklisp's lattice structured top level namespace, which is implemented with a collection of locale objects.

Oaklisp currently runs on the Macintosh ${ }^{\text {TM }}$ personal computer'. It was cross-developed on a lisp machine, where a kernel interpreter was written in Common lisp and the rest of the language was defined in terms of the kernel. $\Lambda$ compiler was written in Oaklisp, urgetting to a stack-nriented bytecode for which an emulator was written on the Macintosh. 'The current memory format uses two low tag bits, thus allowing 30-bit fixnums, pointers, and locatives that can address any word in the logical address space of the 68000 . Characters, weak pointers, and Macintosh handles are represented by 24-bit immediace objects. There are facilities for so-called lightweight processes, and hooks into the window system and Macintosh toolbox, all written in Oaklisp. The current implementation effort has been driven by considerations of simplicity rather than speed. An Oaklisp implementation with efficiency as a primary goal could use all the usual tricks for speeding up lisps. For instance, making cows-parr primitive would specd up list manipulation.

\section{Bibliography}

Harold Abelson ct al.

The Revised Revised Report on Scheme

Al Mcmo 848. MIT Artificial Intcligence Laboratory, 1985.

D. Bobrow, K. Kahn, G. Kiczales, L. Masinter, M. Stcfik, F. Zdybel.

"CommonLoops: Merging Common Lisp and Object-Oriented Programming"

9th International Joint Conference on Artificial Intelligence, 1985

W. Clocksin and C. Mellish.

Programining in Prolog

Springer-Verlag, 1981.

A. Goldberg and D. Robson.

Smalltalk-80: The Language and its Implementation

Addison-Wesley, 1983.

\footnotetext{
SThenks to Bruce Ilorn for his help.
} 
Sonya E. Keene and David A. Moon.

"Flavors: Object-oriented Programming on Symbolics Computers"

Common Lisp Conference, December 1985.

Glenn Krasner, Ed.

Smallialk-80: Bits of History, Wonds of Advice

Addison-Wesley, 1983.

Kevin J. Lang and Barak A. Pearlmutter.

The Oaklisp Language Manual and The Oaklisp Implementation Guide

Unpublished, CMU Computer Science Department, 1985.

D. Moon and D. Weinreb.

The Lisp Machine Manual

Symbolics Inc.

Jonathan A. Rees and Norman I. Adams IV.

"T: a dialect of Lisp or, Lambda: the ultimate software tool"

Proceedings of the 1982 ACM Symposium on Lisp and Functional

Programming. August 1982

Jonathan A. Rees, Norman I. Adams IV, and James R. Mcehan.

The T Manual

Fourth edition, Yalc University Computer Science Department, 1984.

D. Sleator and R. Tarjan.

"Self-adjusting Binary Search Trees"

Proceedings of ISth Symposium on Theory of Computing.1983.

Brian Cantwell Smith

Reflection and Semantics in Lisp

CSLI-84-8. Center for the Study of Language and Information, 1984.

Guy L.cwis Stecle, Jr.

Lambda, the Ullimate Declanative

AI Memo 379, MIT Artificial Intelligence Laboratory. 1976.

Guy Lewis Stecle. Jr. and Gerald Jay Sussman.

Lambda, the Ulitimate Imperative

Al Mcmo 353, MIT Artificial Intelligence Laboratory, 1976.

Guy Lewis Stcelc, Jr. and Gerald Jay Sussman.

The Art of the Interpreter

Al Memo 453, MIT Artificial Intelligence Laboratory, 1978.

G. Sussman, and D. McDermott.

"From PLANNER to CONNIVER - A Genctic Approach"

Proctedings of the Fall Joint Computer Conference. 1972.

AFIPS Press. 